livraisons

d'Histoire

de l'Architecture

\section{Livraisons de l'histoire de l'architecture}

$25 \mid 2013$

Architectures médiévales : types, matières et formes

\title{
La chapelle Saint-Léonard de Rouelles retrouvée (1479-1480) : le vœu d'un seigneur revenu de captivité
}

The Saint-Léonard de Rouelles chapel rediscovered (1479-1480): the vow of a lord back from captivity

Die Wiederentdeckung der Kapelle Saint-Léonard de Rouelles (1479-1480): das

Gelübde eines aus Gefangenschaft heimgekehrten Seigneurs

\section{Mathieu Deldicque}

\section{OpenEdition}

\section{Journals}

Édition électronique

URL : http://journals.openedition.org/lha/304

DOI : 10.4000//ha.304

ISSN : 1960-5994

Éditeur

Association Livraisons d'histoire de l'architecture - LHA

Édition imprimée

Date de publication : 10 juin 2013

Pagination : 55-65

ISSN : 1627-4970

Référence électronique

Mathieu Deldicque, « La chapelle Saint-Léonard de Rouelles retrouvée (1479-1480) : le vœu d'un seigneur revenu de captivité », Livraisons de l'histoire de l'architecture [En ligne], 25 | 2013, mis en ligne le 10 juin 2015, consulté le 19 avril 2019. URL : http://journals.openedition.org//ha/304 ; DOI :

$10.4000 /$ /ha.304

Ce document a été généré automatiquement le 19 avril 2019

Tous droits réservés à l'Association LHA 


\section{La chapelle Saint-Léonard de Rouelles retrouvée (1479-1480) : le vœu d'un seigneur revenu de captivité}

The Saint-Léonard de Rouelles chapel rediscovered (1479-1480): the vow of a lord back from captivity

Die Wiederentdeckung der Kapelle Saint-Léonard de Rouelles (1479-1480): das

Gelübde eines aus Gefangenschaft heimgekehrten Seigneurs

\section{Mathieu Deldicque}

1 La ville du Havre possède un patrimoine médiéval méconnu. Avant même sa fondation par François I ${ }^{\text {er }}$ en 1517, plusieurs édifices, nichés au sein de la seigneurie de Graville et de ses dépendances, dominaient l'estuaire de la Seine. Les seigneurs du lieu, les Malet de Graville, avaient en effet marqué ce territoire de leur empreinte : si le prieuré de Graville et l'église Saint-Michel d'Ingouville constituent les rares vestiges des nombreuses fondations de la famille, la plupart d'entre elles ont malheureusement disparu suite aux troubles révolutionnaires et aux lourdes destructions de l'époque contemporaine.

2 Parmi ces fondations figurait la chapelle Saint-Léonard, située à Rouelles, l'un des fiefs dépendant de la seigneurie de Graville. La redécouverte du compte de construction, d'anciens relevés et d'une sculpture toujours conservée permet de ressusciter ce modeste édifice et d'en comprendre la genèse.

\section{Le commanditaire, Jean VI Malet de Graville}

3 La chapelle fut bâtie à l'initiative de Jean VI Malet de Graville. C'était le fils de Jean V Malet de Gravillle et de Jacqueline de Montaigu, elle-même fille de Jean de Montaigu, grand maitre de l'hôtel de Charles VI. Jean V avait été le premier seigneur de cette vieille famille normande à endosser d'importantes fonctions au service de la Couronne: 
compagnon de Jeanne d'Arc, il fut récompensé de ses services par Charles VII qui le nomma grand fauconnier, panetier et grand maître des arbalétriers de France ${ }^{1}$. Jean VI hérita des seigneuries normandes de son père et des domaines franciliens de sa mère. Suivant l'exemple paternel, il mit son épée au service de Charles VII et devint son chambellan. Le roi l'envoya par la suite en Angleterre pour porter secours à la reine Marguerite d'Anjou, fille du roi René et chef du parti lancastrien dans la guerre des DeuxRoses. Selon les Chroniques d'Engleterre de Jean de Wavrin et un acte du tabellionnage de Rouen $^{2}$, il fut fait prisonnier par les partisans d'Édouard IV vers 1461. Il connut une longue captivité de près de dix-sept ans puisqu'il ne fut de retour en France qu'en 1478. Il reprit aussitôt les rênes de ses domaines, confiés en son absence à son épouse, Marie de Montauban, et à son fils, Louis Malet de Graville, futur amiral de France. En effet, dès son retour, le seigneur entreprit de restaurer la seigneurie familiale de Graville et, notamment, son château ${ }^{3}$. La construction de la chapelle Saint-Léonard de Rouelles s'inscrit dans ce renouveau.

\section{Le chantier de la chapelle de Rouelles d'après les comptes et les documents figurés}

Une ancienne chapelle, Saint-Léonard du Val-Baudry, était attestée depuis le XIV e siècle : les seigneurs de Graville présentaient son chapelain à l'abbaye de Montivilliers, dont elle était dépendante. L'édifice est mentionné dès $1320^{4}$ et plusieurs actes font état du droit de présentation des Malet de Graville au cours du $\mathrm{XV}^{\mathrm{e}}$ siècle ${ }^{5}$. Cette chapelle devait appartenir à la léproserie du même nom attestée jusqu'à l'époque moderne ${ }^{6}$. Sans doute touchée par les raids anglais qui ont ravagé la région durant la guerre de Cent Ans, elle fit l'objet d'une reconstruction complète en 1479-1480.

5 De fait, à peine rentré d'Angleterre, Jean VI finança sur ses propres deniers la totalité de la reconstruction de la chapelle. Le compte de construction conservé aux Archives départementales de la Seine-Maritime permet de reconstituer l'édifice et sa réalisation?. La modestie du chantier a permis de regrouper les dépenses occasionnées sur une seule année comptable, 1479-1480. Le compte comprend ainsi l'ensemble des paiements effectués pour la construction, l'aménagement et l'équipement de la chapelle, depuis la chaux jusqu'au missel. Ces dépenses sont plus ou moins ordonnées : si les frais de gros œuvre sont répertoriés avant ceux engagés pour l'achat du mobilier liturgique, les autres sont mêlés à ceux occasionnés par les travaux effectués concomitamment au château de Graville'.

6 Le maître d'œuvre était Guillaume de Longuesne, maçon demeurant à Harfleur d'après le compte. Ce maçon, recruté par marché, peut-être par Jean Malet lui-même, mais aussi sans doute par monseigneur de Rouville, parent du commanditaire ${ }^{9}$, assisté par le prieur de Graville, du vicomte du lieu et d'autres officiers seigneuriaux, nous est connu par ailleurs. Ainsi, près de dix ans auparavant, en 1470, il fut choisi par la municipalité de Harfleur pour la construction de son hôtel commun ${ }^{10}$. De même, il est qualifié, dans un autre acte de la même année, de maçon de la ville de Harfleur ${ }^{11}$. En 1473, il est payé $6 £$ pour avoir refait la voûte de l'arche d'un moulin et pour avoir pavé l'édifice ${ }^{12}$. Le seigneur voulut sans doute s'offrir les services d'une personnalité déjà reconnue dans la région.

D'après les informations livrées par le compte, Guillaume de Longuesne réalisa à Rouelles la maçonnerie d'un bâtiment de plan rectangulaire d'environ 12 mètres de long sur 5 
mètres de large, avec des murs gouttereaux d'une hauteur de 3,80 mètres environ. L'édifice était couvert de tuiles, possédait deux portes, elles-mêmes surmontées d'arcs en claveaux ${ }^{13}$. Quatre petites fenêtres rondes ou oculi en perçaient les murs, deux de chaque côté, sans meneau, surmontées de claveaux ainsi qu'une fenêtre plus importante, à réseau et en pierre de taille. Ces mêmes murs étaient faits de « caillou », c'est-à-dire de moellons de silex ${ }^{14}$, tandis que les angles et les embrasures étaient réalisés en pierre de taille. On utilisa la pierre de Rainville; Guillaume de Longuesne fut d'ailleurs payé 100 sous tournois supplémentaires pour l'avoir employée.

Deux charpentiers participèrent également au chantier: après avoir passé un marché avec le prieur de Graville et le receveur de la seigneurie, Perinet le Roy réalisa la charpente, tandis que Naudin de la Hogue exécuta les deux portes de la chapelle. Par ailleurs, deux forgerons et un serrurier furent employés pour les pièces de fer et autres serrures appliquées aux portes et fenêtres. L'un de ces forgerons venait également d'Harfleur. Quant aux verrières, elles ne semblaient pas être particulièrement riches, ni historiées : n'est en effet répertorié qu'un seul paiement pour deux verrières « des bouts de l'autel », désignant probablement deux des oculi qui devaient éclairer le chœur. La grande verrière qui ornait vraisemblablement le chevet, nous y reviendrons, n'est mentionnée qu'à propos du plâtre qui servit à la "plastrer »; aucune indication n'est livrée quant à son apparence.

9 Pour pallier ce manque de précisions concernant un édifice aujourd'hui disparu, nous pouvons avoir recours à d'anciennes descriptions qui corroborent les informations glanées dans le compte. On sait en effet que la chapelle fut vendue en 1790 comme bien national, avec la maladrerie qui l'accompagnait ${ }^{15}$. En 1877, elle était habitée par un cultivateur et était décrite en ces termes :

« La chapelle, sans les additions qu'on y a faites, mesure environ 12 mètres de long sur 5 de large. La muraille est épaisse. On reconnaît encore la baie de la fenêtre du chevet. Les deux murs latéraux étaient percés de quatre fenestrelles en partie rebouchées ou détruites. Sur la porte d'entrée, qui se trouvait au bas de la chapelle et sur le côté qui fait face à Frileuse ${ }^{16}$, on voit encore une pierre blanche qui a dû supporter un écusson ${ }^{17}$. »

Un relevé de Roessler, daté de 1860 environ et conservé à la Bibliothèque municipale du Havre, témoigne de cet état. 
Dessins de l'ancienne chapelle Saint-Léonard de Rouelles par Roessler,

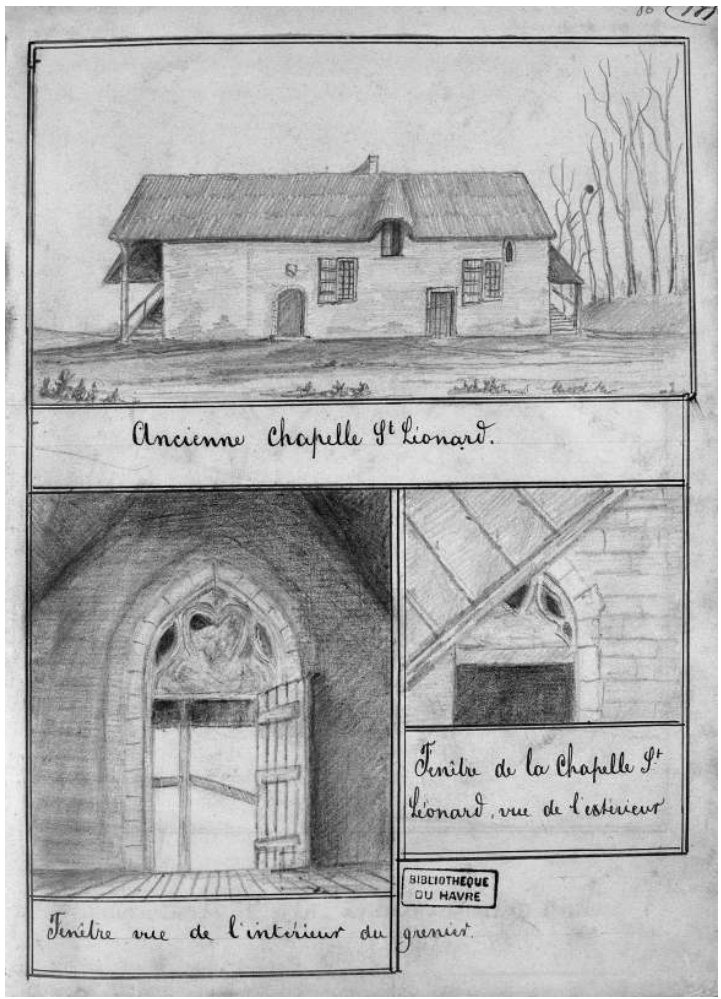

Vers 1860 (Bibliothèque municipale du Havre)

Cl. Kollmann @ 2005 Inventaire général du Patrimoine culturel Région Haute-Normandie

11 La chapelle y apparaît après avoir été transformée en habitation: vraisemblablement divisée en deux niveaux par un plancher, elle était flanquée à l'ouest et à l'est par deux escaliers hors œuvre. La grande baie du chevet, au réseau flamboyant fait de courbes et contrecourbes, existait toujours et éclairait le second niveau. En revanche, on ne perçoit pas sur ce dessin les oculi sans meneau, sans doute comblés à l'époque, mais on remarque la porte latérale en plein-cintre, surmontée de claveaux de pierre de taille et d'un écu dont les armoiries ne sont pas précisées. Une description de 1922 signale qu'il s'agissait des armoiries des Malet de Graville ${ }^{18}$. Sur une photographie datée de cette même année (collection particulière) prise avant la destruction de l'édifice, survenue à la fin des années 1960, on distingue toujours cette porte, tout comme les parements faits de moellons qui composaient la bâtisse. 


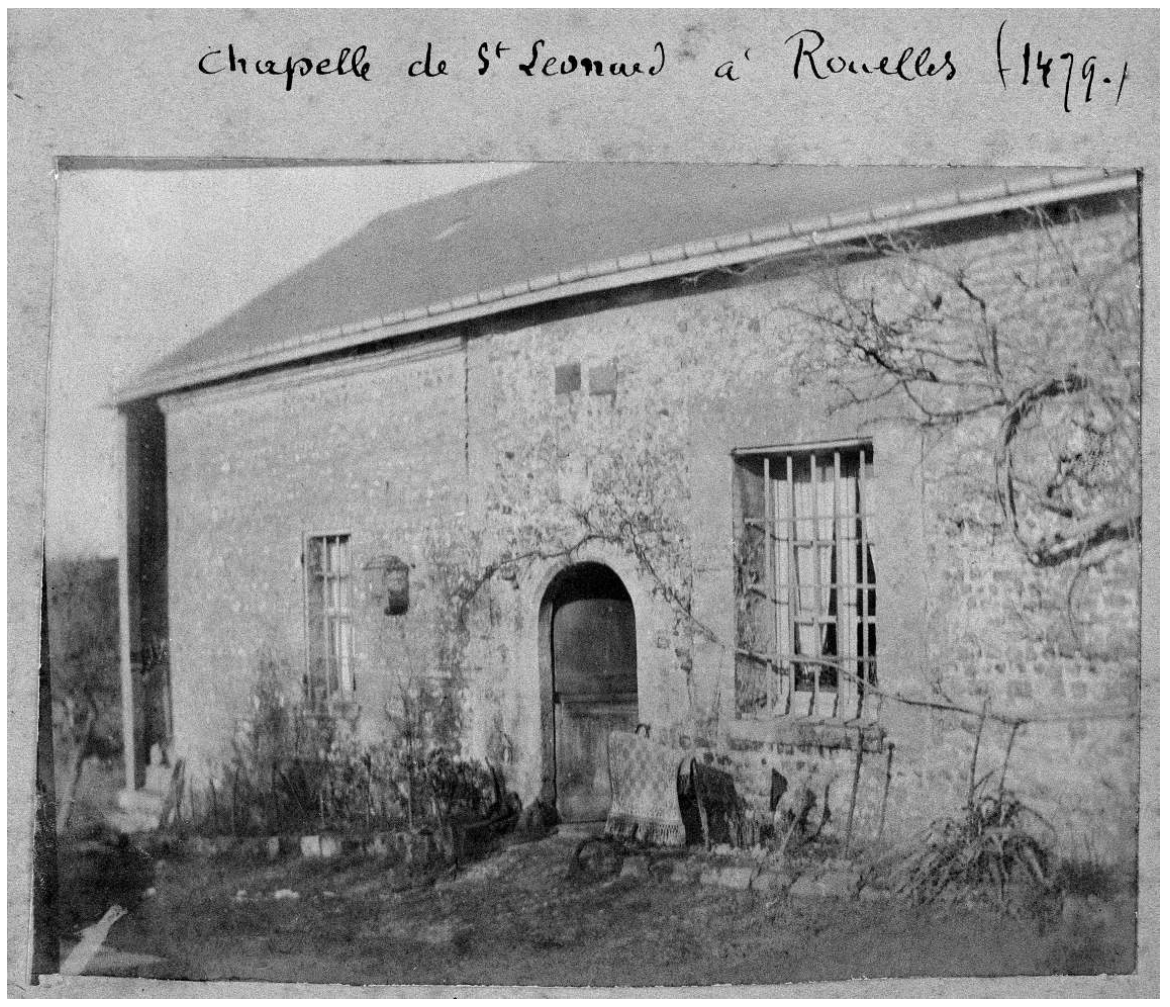

1922 (collection particulière)

Cl. Kollmann (c) 1992 Inventaire général du patrimoine culturel région Haute-Normandie

\section{La statue de saint Léonard}

Le mobilier liturgique et la décoration intérieure furent réalisés et payés la même année. D'après les comptes, les murs de l'intérieur de l'édifice étaient enduits d'un mélange de plâtre, de chaux et de sable, appliqué par un plâtrier et un second maçon, dénommé Jehan Chevalier. Le maître d'œuvre, Guillaume de Longuesne, réalisa pour sa part un autel avec «table et contretable garnie de mouleure tout alentour» ainsi qu'une piscine liturgique que le texte situe "au bout du chancel», c'est-à-dire au bout du chœur liturgique. Quant à l'équipement de la chapelle, rien ne fut oublié, depuis les nappes d'autel, jusqu'aux vêtements que revêtait l'officiant, en passant par l'orfèvrerie liturgique, le coffre pour la ranger et les frais pour bénir l'ensemble.

Fait plus intéressant, la chapelle était décorée d'une statue de bonne qualité dont nous avons retrouvé la trace. On passa, pour 100 sous, un marché avec Guillaume le Franc, "machon et ymaiginier», pour réaliser «ung ymage de pierre de Caen de la remembrance de saint Lynart de cinq piez de long ». Ce marché fut passé devant témoins, dont le prieur de Graville et le vicomte du lieu. Guillaume le Franc était un maitre maçon assez actif en Normandie : si nous ne sommes pas certain que ce soit lui qui figura comme clerc en 1459 à Harfleur lors d'un jugement de la cour ecclésiastique de Montivilliers après une rixe ayant entrainé un accident mortel ${ }^{19}$, nous savons que, plus tard, il œuvra comme maître maçon à l'église Saint-Martin de la même ville et y répara des chapelles ${ }^{20}$ (il est ainsi repéré en 1477 dans un mandat de paiement des officiers municipaux ${ }^{21}$ ). 
L'église Saint-Martin de Harfleur était alors l'un des grands édifices flamboyants de la vallée de la Seine ${ }^{22}$; cependant, ce n'est que vers 1500 que la fabrique y entreprit de grands travaux. Guillaume le Franc intervint donc dans une période de «relative inactivité ${ }^{23}$, pendant laquelle seules quelques réparations de fenêtres et de chapelles furent réalisées. Nous ignorons toutefois si ce fut encore lui qui dirigea l'important chantier au cours duquel, vers 1500, furent érigé l'imposant clocher et agrandies les parties orientales de l'église. Quoiqu'il en soit, avant cette date, en 1490, Guillaume le Franc est repéré à nouveau comme maître d'œuvre de l'église Saint-Michel de Pontl'Évêque : il réalisa les parties hautes de la nef ${ }^{24}$. Il était alors qualifié de maître maçon rouennais et était venu de la capitale normande avec un verrier, Alexandre Dubois, qui avait lui-même œuvré aux côtés de Jean Barbe sur le chantier du château de Gaillon ${ }^{25}$. On le retrouve enfin le 12 septembre 1502 parmi les experts choisis par la ville de Rouen pour une visite du chantier du palais de justice ${ }^{26}$.

La statue réalisée par Guillaume le Franc est aujourd'hui présentée dans l'église SaintJulien du Havre ${ }^{27}$.

Statue de saint Léonard, église Saint-Julien du Havre

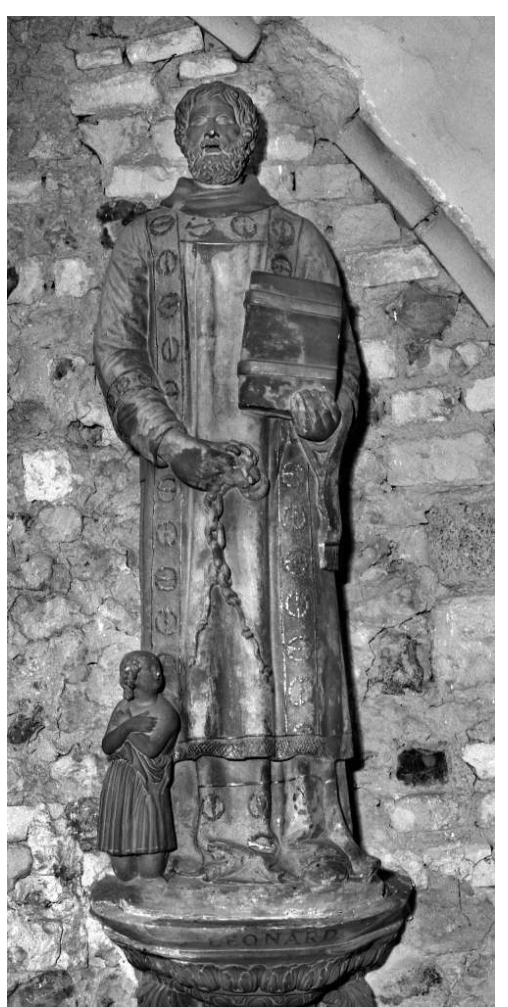

Calcaire, h. $145 \mathrm{~cm}, \mathrm{l} .35 \mathrm{~cm}$.

Cl. Miossec (@ 2002 Inventaire général du patrimoine culturel région Haute-Normandie

Il s'agit du seul témoignage conservé du talent de ce maçon et tailleur d'images polyvalent qui n'hésitait pas, du moins au début de sa carrière, à s'aventurer dans le domaine de la sculpture figurée. Auparavant datée du XVII ${ }^{e}$ siècle ${ }^{28}$, la comparaison de l'œuvre avec le compte ne fait désormais pas de doute sur son attribution et sa datation. Il s'agit d'une statue en ronde bosse de $145 \mathrm{~cm}$ de hauteur, $35 \mathrm{~cm}$ de largeur et $34,5 \mathrm{~cm}$ de profondeur. La hauteur correspond donc tout à fait à celle de cinq pieds mentionnée dans le compte. L'œuvre est réalisée en calcaire (pierre de Caen) et est recouverte d'une 
polychromie déposée par couches successives au cours des siècles. Son revers plat indique qu'elle fut réalisée pour être adossée à une paroi. Elle figure aujourd'hui sur un culot sculpté mais nous ignorons si elle était placée sur un tel culot à l'origine, dans la chapelle de Rouelles.

16 L'œuvre représente saint Léonard, debout et habillé en prêtre : le saint porte une longue aube sous une chasuble dont le bord inférieur est décoré de broderies. Son cou est entouré d'un amict. L'élément le plus intéressant pour notre propos est l'étole décorée de tout son long par des fermaux, tout comme le bas de sa tunique, elle aussi brodée de ces mêmes fermaux : le seigneur de Graville affichait de cette manière ses armes (de gueules à trois fermaux d'or) sur une statue qu'il avait entièrement financée et qui était destinée à devenir l'objet de prières, voire de pèlerinages. La présence de ces meubles héraldiques dut sans doute faire l'objet d'une indication dans le contrat - disparu - passé entre le commanditaire et l'artiste.

Le saint tient dans sa main gauche un livre imposant, visiblement entouré de grosses lanières ou de fermoirs. De cette même main pend un manipule, signe que saint Léonard portait ici les ordres majeurs. Il tient de sa main droite des chaines rompues et à ses pieds figure un personnage, à la petitesse disproportionnée. Ses mains sont croisées sur sa poitrine en signe d'affliction et son regard est tourné vers le saint avec un air d'imploration. Son visage est poupin ; sa bouche entrouverte, son nez petit, ses cheveux bouclés, ses yeux et ses sourcils sont finement sculptés. Cette figure représente vraisemblablement un captif délivré de ses chaînes adoptant une attitude de prière et d'adoration à l'égard de son bienfaiteur. En effet, d'après la vie du saint et la Légende dorée de Jacques de Voragine, Léonard, baptisé par saint Rémi vers 500, obtint de Clovis le droit de délivrer tous les prisonniers qu'il jugeait dignes de cette grâce. Il s'installa par la suite en Aquitaine, à Noblat, où il réalisa nombre de miracles. On le priait au Moyen Âge pour la libération des captifs : cette dévotion fut sans doute partagée par le commanditaire, Jean VI Malet de Graville, qui, comme on l'a dit, avait été détenu en Angleterre et put alors faire le vœu de construire, s'il était libéré, une chapelle en l'honneur de saint Léonard, protecteur des prisonniers. La proximité entre la date du retour du seigneur et celle de la commande de l'édifice et de la statue traduit bien la préoccupation qu'il eut à exécuter son vœu, rappelé par la présence des fermaux sur les habits du saint.

La figure expressive du petit captif contraste avec le hiératisme de Léonard: vu de face, le corps de ce dernier forme un bloc d'où peu d'éléments dépassent. Sa tête est un peu trop petite par rapport au reste du corps. Les traits de son visage sont fortement marqués, le nez fort, les yeux grand ouverts, les oreilles bien dessinées, la bouche entrouverte; ce style ne correspond pas à celui du visage du captif. Il nous est difficile de faire des rapprochements avec d'autres statues dans une région, les alentours du Havre, qui a perdu une grande partie de son patrimoine médiéval au cours de la Seconde Guerre mondiale. Il n'en reste pas moins que cette statue de saint protecteur, destinée à être un support pour les prières de paroissiens voire de pèlerins, peut s'insérer dans le groupe des statues de saints protecteurs et guérisseurs qui fleurirent dans la Normandie de la fin du Moyen Âge ${ }^{29}$. Ces saints revêtent, comme saint Léonard à Rouelles, les riches costumes ecclésiastiques de l'époque, rehaussés d'orfrois et de brocarts. Ainsi, les statues de saint Nicolas et de saint Philibert, installées dans l'église de Saint-Philbert-sur-Risle (Eure), peuvent soutenir la comparaison avec celle de saint Léonard, leur contemporaine. Ces deux statues, frontales, sont elles aussi placées sur des dais. Les «nouvelles valeurs plastiques de la statuaire normande d'après $1470 »^{30}$ apparaissent dans les trois statues 
qui nous intéressent : leur port est altier, leur expression froide et austère, leur chasuble très richement ornée de brocarts et leur maintien statique ${ }^{31}$. Guillaume le Franc s'inscrivait dans le courant stylistique de la sculpture de la fin du XV siècle qui s'était répandu depuis Rouen, ville dont il était d'ailleurs originaire.

Si la chapelle de Rouelles était, somme toute, un édifice assez modeste et relativement peu coûteux ${ }^{32}$, son commanditaire fit toutefois appel à des artisans qualifiés et notables de la région (notamment du port voisin de Harfleur où les métiers du bâtiment purent se développer à la faveur des grands chantiers qui y étaient entrepris), assistés épisodiquement par une main-d'œuvre locale pour les opérations demandant peu de qualification. De même, malgré son apparence frustre, elle était décorée avec soin et bien équipée. En témoigne le «messel de papier en moulle ", c'est-à-dire un missel imprimé, qui lui fut destiné. Beaurepaire parle, à ce propos, de «l'apparition du premier missel imprimé, à l'usage des églises du diocèse » avec un « prix relativement élevé » (4 livres 5 sous $^{33}$. En effet, l'apparition de l'imprimerie fut tardive à Rouen : ce n'est qu'en 1485 que parut le Prologue de l'entrée du roi Charles VIII, première œuvre à sortir des presses rouennaises ${ }^{34}$. C'est donc probablement à Paris que Jean VI s'était procuré ce missel imprimé : c'est bien la preuve qu'il voulait doter sa nouvelle fondation des dernières nouveautés.

Il ne faut donc pas s'arrêter sur la grande simplicité des partis architecturaux qui concernent Saint-Léonard de Rouelles. Cette simplicité correspond à la fois à son usage et à son statut de chapelle rattachée à une petite maladrerie rurale. Réalisation probable d'un vœu formulé lors de sa captivité, l'édifice était également pour Jean VI Malet de Graville un moyen de reprendre en main ses domaines, de réaffirmer son emprise après une longue absence. Il appartenait ainsi à tout un réseau progressivement mis en place de chapelles et d'églises qui, issues de fondations effectuées par la famille qui possédait le droit de présentation à leur cure, émaillaient ses seigneuries et furent presque toutes reconstruites dans la seconde moitié du XV siècle : l'église Saint-Michel d'Ingouville ${ }^{35}$, la chapelle Sainte-Marie-Madeleine de la Couldre à Harfleur ${ }^{36}$, la chapelle Sainte-Anne de Fontaine $^{37}$. Ce quadrillage systématique, effectué notamment grâce à un tissu de petites chapelles, est d'ailleurs caractéristique de la reconstruction de bien des seigneuries rurales après la guerre de Cent $\mathrm{Ans}^{38}$.

\section{ANNEXES}

Annexe : Comptes de la seigneurie de Graville, extraits concernant les dépenses de construction et d'aménagement destinées à la chapelle Saint-Léonard de Rouelles

1479-1480. - Graville

Arch. dép. Seine-Maritime, A 7, extraits ${ }^{39}$.

Cuvres et reparacions 
1. [Fol. 1] A Guillaume de Longuesne, machon demourant a Harefleu, pour avoir fait de machonnerie, trouver les matieres et rendues en place la chapelle Saint Lynart sur Rouelle contenant 40 piez de long et 22 de large ou environ ainsi qu'il ensuit, c'est assavoir l'enclos de lad. chapelle de caillou, les costez de 12 piez de hault entablez ${ }^{40}$ et les pignons amortis ${ }^{41}$ a leur raison et enchapez ${ }^{42}$, deux huisseries voultees de pierre de taille par dehors et par dedans, quatre fenestres es deux costés toutes rondes, sans maignel ${ }^{43}$, voultees de pierre de taille, une fenestre sur un maingnel faicte en formement, bien et gentement taillee et voultee par dehors et par dedans de pierre de taille, ung autel, la table et contretable garnie de mouleure tout alentour, une pichine au bout du chancel bien gentement faicte et les coings de lad. chapelle fais semblablement de pierre de taille, $59 £ 10 \mathrm{~s}$. de laquelle somme a esté paié par le chapelain $20 £$, demeure cy : $39 £ 10 \mathrm{~s}$.

2. Item pour le vin du marchié : $10 \mathrm{~s}$.

3. Item donné aud. Guillaume par monseigneur de Rouville, aprés l'advis eu du prieur de Graville, du viconte et autres officiers de lad. seigneurie, pour certaines pertes que led. machon a eues en faisant led. ouvrage et aussi consideré qu'il a fait tout ce que est fait a lad. machonnerie de pierre de taille de pierre de Rainville et il ne le devoit faire sinon de pierre de Caen qui eust esté grant dommaige pour lad. chapelle : 100 s.t.

4. [Fol. 1v] A Perinet le Roy, charpentier, pour avoir fait l'ouvrage de charpenterie au comble de lad. chapelle, trouvé le boys et a ses despens comme il ensuit, c'est assavoir trois trefs ${ }^{44}$ et trois montants, doubles sablieres enjouee tout a l'entour, trois quevrons ${ }^{45}$ a la latte et chacun quevrons une jambette ${ }^{46}$ a tenon par dessoubz et dessus atachees a clou, par marchié fait avec lui par le prieur de Graville et le receveur en la presence de plusieurs gens de Rouelle : $13 £ 10 \mathrm{~s}$.

5. Item pour le vin du marchié : $10 \mathrm{~s}$.

6. A Naudin de la Hogue, charpentier, pour sa paine d'avoir fait les deux huys servans a lad. chapelle, enchassillez ${ }^{47}$ et trouvé le boys : $22 \mathrm{s.} 6 \mathrm{~d}$.

7. A Henry Douchelin, mareschal, pour 4 gons a pendre lesd. huys : 3 s. $6 \mathrm{~d}$.

8. A $(. . .)^{48}$, serrurier, pour quatre vertevelles ${ }^{49}$ traversans lesd. huys, deux touroul $x^{50}$ aud. grant huys et une clere au petit huys par marché fait avec luy : $15 \mathrm{~s}$.

9. Item pour une serrure de boys aud. petit huys : $3 \mathrm{~s} .9 \mathrm{~d}$.

10. A Jehannin le Mareschal, demourant a Harefleu, pour avoir fait la feraille mise a la grant verriere de lad. chapelle et es 4 petites, le tout pesant 99 livres de fer, a 9 d. la livre, vallent : $66 \mathrm{s.} 9 \mathrm{~d}$.

11. Item a esté despensé par les charretiers qui ont admené le boys du Tomble a lad. chapelle, la pluspart de 5 lieues l'ung et l'autre partie de Blienes par plusieurs fois : $20 \mathrm{~s}$.

12. [Fol. 2] Item pour huit milliers de tuille a couvrir lad. chapelle pour chacun millier rendu sur le lieu avec les festiers ${ }^{51} 40$ s. t., pour ce : $16 £$

13. Item pour 1300 et demy de late ${ }^{52} \mathrm{du}$ pris de $2 \mathrm{~s}$. t. le cent, pour ce : $27 \mathrm{~s} .3 \mathrm{~d}$.

14. Item pour ${ }^{53}$

15. Item pour 6 milliers 600 de clou a latte pour chacun millier 9 blans, pour ce : $24 \mathrm{~s} .10 \mathrm{~d}$.

16. Item pour ung cent de clou a demy tillac ${ }^{54}$ a clouer les jambettes es quevrons : 2 s. 6 d. 
17. Item pour ung quarteron de caux ${ }^{55}$ a couvrir lad. chapelle : $18 \mathrm{s.} 9 \mathrm{~d}$.

18. Item pour trois blenees ${ }^{56}$ de sablon, pour chacun bleuee $22 \mathrm{~d}$. sont : $5 \mathrm{~s} .6 \mathrm{~d}$.

19. Item a Germain le Plastrier pour sa paine d'avoir couvert lad. chapelle, par marchié fait avec lui par led. prieur de Graville et led. receveur : $70 \mathrm{~s}$.

20. Item donné au serviteur dud. Germain lequel a couvert lad. chapelle : $22 \mathrm{~d}$.

21. Item a esté donné par led. receveur a plusieurs compaignons qui ont vuidé lad. chapelle et rabessié les terres demourant et qui ont aidé a mettre le tuille en hault : $10 \mathrm{~s}$.

22. Item pour les deux verrieres des bous de l'autel : 16 s. 6 d.

23. Item pour 4 livres de plastre a plastrer la grant verriere, pour chacun boisseau $2 \mathrm{s.}$ sont : $8 \mathrm{~s}$.

24. Item a Guillaume Barete pour avoir employé led. plastrier : 2 s. 9 d.

[Fol. 2v-4] $]^{57}$

25. [Fol. 4v] A Guillaume le Franc, machon et ymaiginier, pour avoir fait a lad. chapelle de Saint Lynart ung ymage de pierre de Caen de la remembrance de saint Lynart de cinq piez de long et trouvé lad. pierre, par marchié fait avec lui, presens le prieur de Graville, le viconte dud. lieu de Graville, maistre Nicolle le Jeune et autres : $100 \mathrm{~s}$.

26. A Germain le Plastrier et Jehan Chevalier, machon, pour avoir enduit lad. chappelle, trouvé le plastre, la caux et le sablon : $6 £$

27. Item ung autel beneist pour lad. chapelle : $5 \mathrm{~s} .6 \mathrm{~d}$.

28. En sept aulnes et demie de toille pour faire trois touailles d'autel a service lad. chapelle et pour la facon d'icelles : 29 s. 4 d.

29. Item pour beneir touailles : $11 \mathrm{~d}$.

30. Item en 4 aulnes de toille pour faire une aube et ung amyt pour servir a icelle chapelle : 7 s. $6 \mathrm{~d}$.

31. Item ung casuble de couleur vermeil, fanon et estolle de mesmes et ung chaint ${ }^{58}$ pour service a lad. chapelle : $40 \mathrm{~s}$.

32. Item pour beneir lad. aulbe, amyt, casuble, estolle et fanon : $22 \mathrm{~d}$.

33. Item pour ung calice d'estain : $5 \mathrm{~s}$.

34. Item pour ung messel de papier en moulle ${ }^{59}: 4 £ 5 \mathrm{~s}$.

35. Item pour beneir led. calice d'estain: $11 \mathrm{~d}$.

36. Item pour ung estuy a corporeaulx : 7 s. $4 \mathrm{~d}$.

37. Item pour deux chopines : 3 s. $8 \mathrm{~d}$.

38. Item pour ung coffre servans a lad. chapelle pour mettre lesd. ornements dessusd. : 15 s.

Somma hujus capituli $142 £ 19$ s. 3 d. 


\section{NOTES}

1. Père Anselme, Histoire généalogique et chronologique de la maison royale de France, des pairs, etc., Paris, t. VIII, 1733, p. 86-87.

2. Acte édité dans Paul-Michel Perret, Notice biographique sur Louis Malet de Graville, amiral de France (144 ?-1516), Paris, 1889, p. 243-244.

3. Pour l'étude du château de Graville, notamment à partir des comptes conservés (Archives départementales de la Seine-Maritime, A 7), se reporter à notre thèse d'École des chartes : Entre Moyen Âge et Renaissance? La commande artistique de l'amiral Louis Malet de Graville (v. 1440-1516), thèse pour le diplôme d'archiviste paléographe, 2012, t. 1, p. 182-188.

4. Arch. dép. Seine-Maritime, G 5346. Voir également Charles de Beaurepaire, « La chapelle SaintLéonard de Rouelles ", dans Bulletin de la Commission des Antiquités de la Seine-Inférieure, 1877, p. $165-166$.

5. Arch. dép. Seine-Maritime, 54 H 472.

6. Ibid. Voir également le Dictionnaire topographique du département de Seine-Maritime, Paris, C.T.H.S., t. II, 1984, p. 913.

7. Arch. dép. Seine-Maritime, A 7. Se reporter à l'édition qui figure en annexe de cet article.

8. Les lignes de compte concernant les travaux du château de Graville ne figurent pas en annexe. Voir Deldicque, 2012, vol. 2, p. 75-81.

9. Il s'agit sans doute de Guillaume de Rouville († 1492), conseiller et chambellan de Louis XI, qui avait épousé la sœur de Jean VI Malet de Graville, Louise. Cf Père Anselme 1733, t. VII, p. 710-711.

10. Beaurepaire 1877, p. 169.

11. Ibid.

12. Procès-verbaux de la Commission départementale des antiquités de la Seine-Inférieure, t. II, Rouen, 1867, p. 172.

13. Le compte mentionne «deux huisseries voultees de pierre de taille par dehors et par dedans ».

14. Philippe Lardin, Les chantiers du bâtiment en Normandie orientale à la fin du Moyen Âge (matériaux et ouvriers), sous la dir. de Jean-Pierre Legay, thèse doctorat, histoire, Rouen, 1995, dactyl., p. 80-81.

15. Notice de la base en ligne Mérimée, référence IA7600020 (rédigée par Claire Étienne).

16. Lieu-dit de la commune de Rouelles.

17. Beaurepaire 1877, p. 166.

18. Notice de la base Mérimée, référence IA7600020.

19. En effet, un certain Guillaume le Franc fut jugé avec un autre clerc, Colin Langlois, pour avoir proféré des injures à l'égard d'un jeune homme armé, vassal de l'écuyer Robert du Sel, à cause d'un porcelet, et pour s'être battu contre lui sur les murs de Harfleur, à la nuit tombée. Au cours de la rixe, le vassal tomba et décéda après avoir heurté un puits de bois, ce qui amena les deux clercs à être jugés par la cour ecclésiastique. Néanmoins, seul Colin Langlois écopa d'une amende. Voir Arch. dép. Seine-Maritime, G 5269, registre des expéditions de la cour ecclésiastique de Montivilliers (1424-1464): "Anno Domini $\mathrm{M}^{\circ} \mathrm{CCCC}^{\circ} \mathrm{LIX}^{\circ}$, die jovis ante festum Sancti Bartholomei, Guillermus Le Franc et Colinus Langlois, clerici, fecerunt emendam super eo quod, post plura verba contumeliosa, habita cum quodam juvene armato, clientulo Roberti du Sel, scutiferi, ratione et ad causam cujusdam porcelli, ipsum clientulum de dicto porcello percusserunt pluries [...] et, adveniente nocte, ipsis circa horam decimam de nocte vigillias noctis facientibus supra muros ville Hareflotti, alter ipsorum hurtavit seu butavit de subtus muros ad terram subtus, adeo et taliter quod (clientulus) cecidit supra quemdam puy gallice de ligno, a quo fuit quasi letaliter percussus. Taxata pro Colino Lenglois, LX solidi. » 
20. Arch. dép. Seine-Maritime, G 6876 et 3E6/120, nº 64 (Cité dans Philippe Lardin, Les chantiers du bâtiment en Normandie orientale à la fin du Moyen Âge (matériaux et ouvriers), thèse de doctorat, sous la dir. de Jean-Pierre Leguay, Univ. Rouen, 1995, dactyl., p. 333).

21. Florian Meunier, L'architecture flamboyante dans la vallée de la Seine, de Vernon à Harfleur, thèse de doctorat, sous la dir. de Dany Sandron, Univ. Paris IV-Sorbonne, 2009, 2 vol. , dactyl., p. 419.

22. Sur cette église, voir Meunier 2009, p. 417-423.

23. Meunier 2009, p. 419

24. Arch. dép. Calvados, G 1164.

25. Les vitraux de Basse-Normandie, Corpus Vitrearum Medii Aevii, 8, sous la dir. de Martine Callias Bey et Véronique David, Rennes, Presses Universitaires de Rennes, 2006, p. 98.

26. M. de Stabenrath, Le palais de justice de Rouen, Rouen, Édet, 1842, p. 80-81. Voir également Marius Vachon, La Renaissance française : l'architecture nationale, les grands maîtres maçons, Paris, E. Flammarion, 1910, p. 114.

27. Alors que la statue avait été placée, lors de la désaffectation de la chapelle à la Révolution, à l'extérieur de l'édifice, elle fut transférée en 1852 à Saint-Julien du Havre, non sans se faire prier : la tradition rapporte qu'on dut réciter un Pater et un Ave pour qu'elle daignât se faire transporter (Article du Petit Havre, n 968, février 1943)!

28. Base Palissy, référence IM76004417. Nous tenons à remercier Mme Claire Étienne, conservateur général du patrimoine, chef du service Inventaire et patrimoine, région HauteNormandie, ainsi que les services de l'Inventaire de Haute-Normandie de nous avoir aidé dans la redécouverte de cette sculpture.

29. Chefs-d'œuvre du gothique en Normandie: sculpture et orfèvrerie du XIII ${ }^{e}$ au XV $\mathrm{XV}^{e}$ siècle, sous la dir. de Catherine Arminjon et Sandrine Berthelot, Milan, 5 continents, 2008, p. 95-96.

30. Ibid., p. 96.

31. Pour une notice de la statue de saint Philibert, voir Chefs-d'œuvre du gothique en Normandie, 2008, n50, p. 168-169.

32. L'ensemble, auquel furent ajoutées les nombreuses dépenses de réfection du château de Graville, coûta 142 livres, 19 sous et 3 deniers (Arch. dép. Seine-Maritime, A 7).

33. Beaurepaire 1877, p. 169.

34. Catalogues régionaux des incunables des bibliothèques publiques de France, vol. XVII, HauteNormandie, par Valérie Neveu, Genève, Droz, 2005, p. 8.

35. Deldicque, 2012, t. 1, p. 237-240.

36. Arch. dép. Seine-Maritime, G 5343.

37. Arch. dép. Seine-Maritime, G 9489.

38. Ainsi, à la même époque, Pierre le Gendre quadrilla lui aussi ses seigneuries du Vexin par ses fondations ecclésiastiques : la chapelle du Boisgeloup, les églises de Parnes et de Magny (Étienne Hamon, Un Chantier flamboyant et son rayonnement : Gisors et les églises du Vexin français, Besançon, Presses universitaires de Franche-Comté, 2008, p. 66-67).

39. Seules sont ici éditées les lignes comptables correspondant au chantier de la chapelle SaintLéonard de Rouelles. Sont indiquées en note les significations des mots spécifiques au domaine de la construction à la fin du Moyen Âge ou à la région. Pour ce faire, ont été mis à contribution Le Dictionnaire du Moyen Français (http://atilf.fr/dmf) et la thèse de Philippe Lardin (Lardin 1995).

40. Les murs gouttereaux sont terminés par un chaîneau ou une corniche.

41. Les rampants des pignons sont moulurés.

42. Pourvus d'un larmier saillant.

43. Meneau, remplage de baie.

44. Pièces de bois horizontales qui traversaient toute la largeur de l'édifice et formaient les poutres du plafond sur lesquelles reposaient les solives.

45. Chevrons.

46. Pièce de bois soutenant les chevrons ou les arbalétriers. 
47. Entourés d'un cadre.

48. Passage laissé en blanc.

49. Anneaux qui tiennent le verrou d'une porte.

50. Verrous.

51. Tuiles cintrées, parfois ornées qui couvrent le faîte d'un édifice.

52. Longue pièce de bois clouée aux chevrons, étroite et plate, utilisée en charpenterie.

53. Passage laissé en blanc.

54. Dans la région d'Harfleur et de Montivilliers, les clous à "tillac ", en principe destinés à la construction navale, étaient utilisés sur les chantiers (Lardin 1995, p. 246).

55. Chaux.

56. Le véhicule employé pour le transport du sable était le bennel, à tel point que l'unité couramment utilisée était la bennellée ou blenée de sablon (Lardin 1995, p. 151).

57. Ces folios sont consacrés aux dépenses occasionnées par les travaux effectués au château de Graville et à ses étables. Pour leur édition, voir Deldicque, 2012, vol. 2, p. 75-81.

58. Ceinture.

59. Caractères d'imprimerie.

\section{RÉSUMÉS}

Le passé médiéval de la ville du Havre est méconnu. La redécouverte d'un compte de construction, d'anciens relevés et d'une statue permettent de mettre en lumière l'action des seigneurs du lieu, les Malet de Graville, et de retrouver la trace d'un patrimoine disparu, la chapelle Saint-Léonard de Rouelles. À ce chantier, au parti architectural modeste, contribuèrent quelques artisans notables, notamment le maître d'œuvre, Guillaume de Longuesne, ou encore le maçon et tailleur d'images Guillaume le Franc, auteur de la statue de saint Léonard, patron des prisonniers, qui ornait l'édifice. Cette sculpture au décor héraldique éloquent ainsi que la date de construction de la chapelle font d'elle le fruit d'un vœu de Jean VI Malet de Graville formulé durant sa captivité anglaise et la marque de sa volonté de reprendre en main des seigneuries ravagées par la guerre de Cent Ans et délaissées pendant son absence.

The medieval past of the city of Le Havre is not well known. The rediscovery of a construction account, old drawings and a statue has enabled us to highlight the action of the Malet de Graville lords and to trace a lost heritage, the Saint-Léonard de Rouelles chapel. This modest building was created by some notable craftsmen, including the mason Guillame de Longuesne or the mason and sculptor Guillaume le Franc who made the statue of Saint Léonard, patron saint of prisoners, which adorned the chapel. This sculpture decorated by the heraldic ornaments and the date of construction of the building show that it was probably the consequence of a vow made by Jean VI Malet de Graville during his captivity in England. It is also the sign of his resolve to regain control over his lands torn by the Hundred Year War, and neglected during his absence.

Die mittelalterliche Geschichte von Le Havre wird oftmals verkannt. Die Wiederentdeckung von Baurechnungen, alten Aufzeichnungen sowie einer Statue hat es nun erlaubt, die Tätigkeit der lokalen Herrschaft, der Familie Malet de Graville, verstärkt ins Licht zu rücken und auf die Spuren eines verschwundenen Denkmals, der Kapelle Saint-Léonard de Rouelles, aufmerksam zu machen. An diesem architektonisch gesehen bescheidenen Bau waren mehrere bedeutende 
Handwerker beteiligt, insbesondere der Baumeister Guillaume de Longuesne und der Steinmetz und Bildhauer Guillaume le Franc, Schöpfer der den Bau schmückenden Statue des heiligen Leonhard, des Schutzheiligen der Gefangenen. Aus dem reichhaltigen heraldischen Dekor, der Leonhardsskulptur sowie aus der Zeitpunkt des Baus der Kapelle lässt sich erschließen, dass ihre Errichtung einem von Jean VI. Malet de Graville in englischer Gefangenschaft geleisteten Gelübde folgt und seinen Willen zur Machtrückgewinnung in seinen Ländereien verdeutlicht, die durch den Hundertjährigen Krieg verwüstetet und während seiner Abwesenheit verfallen waren.

\section{AUTEUR}

\section{MATHIEU DELDICQUE}

Mathieu Deldicque, né en 1987, est archiviste paléographe. Il a soutenu en 2012 une thèse d'École des chartes sous la direction de Philippe Plagnieux et d'Étienne Hamon : Entre Moyen Âge et Renaissance? La commande artistique de l'amiral Louis Malet de Graville (v. 1440-1516). Il poursuit ses recherches en thèse de doctorat à l'université de Picardie Jules Verne, sous la direction d'Étienne Hamon. Il est l'auteur de plusieurs articles consacrés à cette thématique. Il est actuellement conservateur du patrimoine stagiaire (spécialité musées). Adresse électronique :

mathieu.deldicque@gmail.com 